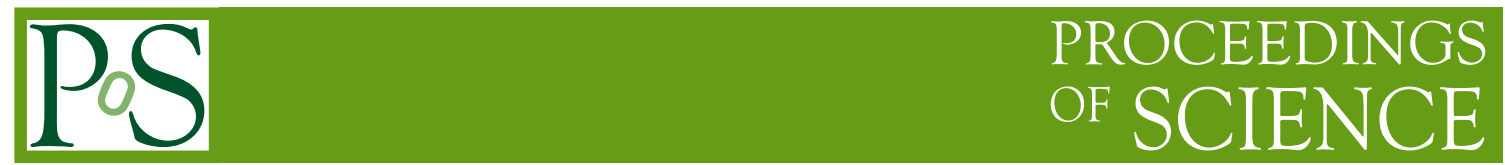

\title{
Search for Muon to Electron Conversion at J-PARC - COMET -
}

\section{Yoshitaka Kuno*i}

Department of Physics, Osaka University

E-mail: kuno@phys.sci.osaka-u.ac.jp

We would like to present the status of the COMET experiment, which aims at a search for muon to electron conversion in a muonic atom with an experimental sensitivity of better than $10^{-16}$ at J-PARC (Japan Proton Accelerator Research Complex), Japan.

35th International Conference of High Energy Physics - ICHEP2010,

July 22-28, 2010

Paris France

* Speaker.

${ }^{\dagger}$ On behalf of the COMET collaboration 


\section{Introduction}

The search for charged lepton flavor violation (CLFV) with muons has large potential to obtain hints for new physics beyond the Standard Model. In the framework of the Standard Model (SM) with massive neutrinos and their mixing, the branching ratio of $\mu \rightarrow e \gamma$ decay can be estimated to be about $10^{-54}$. Therefore, the contribution from the SM with massive neutrinos turns out to be very tiny. As a result, it can be concluded that observation of CLFV would indicate a clear signal of new physics beyond the SM with massive neutrino. Among new physics models beyond the SM, a well-motivated physics model is a supersymmetric (SUSY) model, in particular SUSYOGUT or SUSY-seesaw models. Their predictions are just below the present experimental bounds, and therefore the CLFV search would have a large potential of discovery.

\section{What is $\mu^{-} N \rightarrow e^{-} N$ in a muonic atom ?}

The event signature of coherent $\mu^{-} N \rightarrow e^{-} N$ conversion in a muonic atom is a mono-energetic single electron emitted from the conversion with an energy $\left(E_{\mu e}\right)$ of $E_{\mu e}=m_{\mu}-B_{\mu}-E_{\text {recoil }}$, where $m_{\mu}$ is the muon mass, and $B_{\mu}$ is the binding energy of the $1 s$ muonic atom. $E_{\text {recoil }}$ is the nuclear recoil energy which is small and can be ignored. Since $B_{\mu}$ varies for various nuclei, $E_{\mu e}$ could be different. For instance, $E_{\mu e}=104.3 \mathrm{MeV}$ for titanium (Ti) and $E_{\mu e}=94.9 \mathrm{MeV}$ for lead $(P b)$.

From an experimental point of view, $\mu^{-} N \rightarrow e^{-} N$ conversion is a very attractive process in the following reasons: (1) First, the energy of the signal electron of about $105 \mathrm{MeV}$ is far above the end-point energy of the normal muon decay spectrum $(\sim 52.8 \mathrm{MeV})$. (2) Secondly, since the event signature is a mono-energetic electron, no coincidence measurement is required. The search for this process has the potential to improve sensitivity by using a high muon rate without suffering from accidental background events, which would be serious for other processes, such as $\mu \rightarrow e \gamma$ and $\mu \rightarrow$ eee decays.

The previous search for $\mu^{-} N \rightarrow e^{-} N$ conversion was performed by the SINDRUM II collaboration at PSI. The SINDRUM II spectrometer consisted of a set of concentric cylindrical drift chambers inside a superconducting solenoid magnet of 1.2 Tesla. They set an upper limit of $B\left(\mu^{-}+A u \rightarrow e^{-}+A u\right)<7 \times 10^{-13}$.

\section{The COMET experiment at J-PARC}

A next experimental projects to search for $\mu^{-} N \rightarrow e^{-} N$ conversion with a higher sensitivity are being pursued in Japan is called COMET (Coherent Muon to electron Transition). In the COMET experiment, to suppress background events, in particular beam-related backgrounds, the following key elements have been proposed.

- Beam pulsing: Since muonic atoms have lifetimes of the order of $1 \mu \mathrm{sec}$, a pulsed beam with its width that is short compared with these lifetimes would allow one to remove prompt background events by performing measurements in a delayed time window. To eliminate prompt beam-related backgrounds, proton beam extinction is required during the measurement interval.

- Pion Capture System with a High Solenoid Field: Superconducting solenoid magnets of a high magnetic field surrounds a proton target to capture pions in a large solid angle. This is called the pion capture system. It leads a dramatic increase of muon yields by three orders of magnitude. 
- Curved Solenoids for Muon Transport: The curved solenoids are adopted to select charges and momenta of muons as well as removing neutral particles in a beam. In a curved solenoidal magnetic field, a center of the helical trajectory of a charged particle is shifted perpendicular to the curved plane. The shift, whose amount is given as a function of momentum and its charge, makes a dispersive beam.

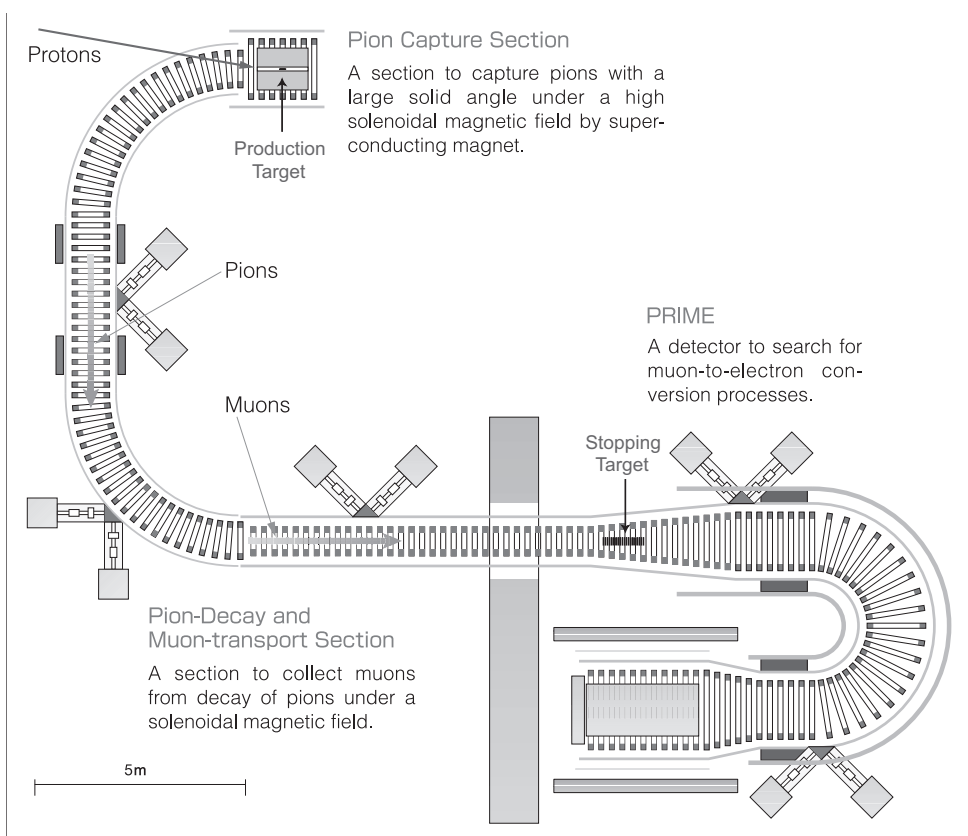

Figure 1: Schematic layout of the COMET experiment at J-PARC. It consists of the pion capture section, the pion-decay and muontransport section, and the detector with curved electron transport solenoids.

The COMET experiment is being proposed at the Japan Proton Accelerator Research Complex (J-PARC), Tokai, Japan. [1] The COMET uses a proton beam of $56 \mathrm{~kW}$ from the J-PARC main ring. The aimed single event sensitivity at COMET is $3 \times 10^{-16}$. A schematic layout of the COMET experiment is presented in Figure 1, The COMET experimental apparatus has several features. One of them is the adoption of $\mathrm{C}$-shape curved solenoid magnets for its muon beamline, and the other is a $e^{+}$curved solenoid spectrometer in COMET. In the muon beam line of the COMET experiment, 180-degree bending solenoids would eliminate high-energy muons which would produce $100 \mathrm{MeV}$ electrons in their decays in flight. Secondary, a curved solenoid electron spectrometer in COMET is useful to eliminate low-energy events from muon decays in orbit before going into the detector, resulting in lower single counting rates in the detectors. The COMET experiment got a stage- 1 approval at J-PARC, 2009 and is working for the final stage-2 approval.

\section{References}

[1] Y. Kuno et al. (COMET collaboration), "A Experimental Search for Lepton Flavor Violating $\mu^{-} N \rightarrow e^{-} N$ Conversion at Sensitivity of $10^{-16}$ with A Slow-Extracted Bunched Proton Beam", J-PARC Proposal, 2007. 